\title{
VANETs Signal Quality-based Route Selection in Smart Cities
}

\author{
Michal Vondra \\ Department of Telecommunication Engineering \\ Czech Technical University in Prague \\ Prague, Czech Republic \\ michal.vondra@fel.cvut.cz
}

\author{
Soufiene Djahel, John Murphy \\ Lero, School of Computer Science and Informatics \\ University College Dublin \\ Dublin, Ireland \\ soufiene.djahel@ucd.ie,j.murphy@ucd.ie
}

\begin{abstract}
In road networks, the most common metrics to determine the optimal route relaying two points are either the path length or the travel time. However, as autonomous smart cars are expected to emerge in future smart cities and lead to an unprecedented growth of mobile applications spectrum for both drivers and passengers, we argue that other metrics can be also considered for route selection. Therefore, the best route for a driver will be also determined by specific in-vehicle users' requirements such as, ensuring sufficient quality for wireless connection with minimum cost, and selecting preferred access networks, etc. To this end, we propose an original algorithm that enables route selection based on maximum expected signal quality with respect to the drivers' maximum tolerated prolongation of journey. Our ultimate objective is to select the route that ensures sufficient signal quality for users on board by maximizing the usage of VANETs resources, through RSUs, while minimizing the usage of the costly LTE-A resources. The obtained simulation results, under several scenarios, have proven the high efficiency of the proposed algorithm.
\end{abstract}

Keywords-VANETs; LTE-A Offloading; Route Selection; Heterogeneous Networks; Smart Cars; Smart Cities;

\section{INTRODUCTION}

In smart transportation system, each vehicle will be able to communicate via Vehicular Ad-hoc NETwork (VANET) with the vehicles in the vicinity or with the fixed infrastructure in order to exchange essential traffic information. Although VANET technology is developed primarily to enable traffic information sharing among vehicles, as well as among vehicles and Road Side Units (RSUs) to improve the driving safety, the on-board users can also avail from this technology for their Internet connection. However, the users' requirements in terms of Quality of Service (QoS) guarantees can be significantly higher than QoS requirements of common road traffic information. Therefore, ensuring sufficient QoS for users in VANETs is one of the main challenges [1] in vehicular networks.

In order to solve this issue, recent research efforts in wireless networks are focused on the potential heterogeneous integration of IEEE $802.11 \mathrm{p}$ protocol, denoted as Wireless Access in Vehicular Environments (WAVE), used in VANETs with the 3GPP cellular networks, such as UMTS or LTE [2], [3], and [4]. The advantages of VANETs are especially the fast adaptation to network topology change, and the application of clustering algorithms to ensure better organization of vehicles within the network. On the other hand, the main advantage of cellular mobile networks is the higher maximum throughput (e.g. in case of LTE-A more than $1 \mathrm{~Gb} / \mathrm{s}$ ) compared to the maximum throughput achievable in VANETs, which is $27 \mathrm{Mb} / \mathrm{s}$. Moreover, the coverage range of LTE-A base stations (eNBs) can be tens of kilometers, while the transmission range of the RSUs is limited to hundreds of meters only.

By considering these pros and cons, the LTE-A seems to be clearly a better choice for vehicles connection. However, other critical factors need to be considered when making the choice such as, the available resources from operator's point of view and the price of connection from user's point of view. In future transportation systems, the communication of hundreds or thousands of smart cars equipped with advanced systems, as presented by Google [5] and OAA [6], or autonomous self-driving cars (introduced by Google [7]) via LTE-A may lead to network overloading and consequently the unavailability of services for other users lacking alternative connection means [8]. Therefore, the LTE-A connection is charged or is limited by Fair User Policy (FUP) for users. On the other hand, the connection via VANETs is completely free of charge. Moreover, since the coverage range of RSUs is limited, the overloading probability of one RSU by hundreds of communicating cars is suppressed.

The ultimate goal of our paper is to ensure sufficient QoS for the on-board users by maximizing the usage of VANETs resources, while minimizing the usage of the relatively valuable LTE-A resources. To this end, we have proposed novel algorithm that allows selecting the optimal route between departure and destination points of a vehicle with respect to the maximum tolerated prolongation of journey set by the user. Since we assume that all users on board are able to use VANETs connection (through IEEE 802.11p interfaces) instead of LTE-A connection (through LTE-A interfaces), this leads to cheaper services for them, while more available resources remain for other LTE-A users. This means, in our case, that LTE-A network is used only as backup when no sufficient QoS is ensured by VANETs connection.

The rest of this paper is structured as follows. Next section gives an overview on the literature. Section III outlines some 
basic yet realistic assumptions needed for our proposal, and describes the proposed route selection algorithm in detail. The simulation environment and parameters are presented in Section IV. In Section V, the evaluation of the proposed algorithm and its comparison with the pioneer approaches in the literature are presented. The last section highlights the major conclusions and outlines future research directions.

\section{RELATED WORK}

Despite the large spectrum of research efforts addressing different issues related to VANETs technology, QoS guarantees for in-vehicles users, and the selection of the most appropriate access technology that fulfils the increasing applications needs in such heterogeneous environment remain a real challenge [1]. Most of existing works address the QoS requirements in VANETs by employing cellular networks such as UMTS or LTE with main focus on providing efficient Cluster Head $(\mathrm{CH})$ or Gateway $(\mathrm{GW})$ selection mechanisms.

Integrating VANETs with LTE network in hybrid architecture has been described in [2]. In this proposal, the vehicles' clusters management is performed centrally by eNB or remote server. The size of a cluster in this architecture is set to at most the transmission range of IEEE 802.11p protocol. The $\mathrm{CH}$ vehicle which also plays the role of $\mathrm{GW}$ for communication with eNB is selected based only on its accessibility to other vehicles in the same cluster. This means that the vehicle directly reachable by most of the cluster members is elected as $\mathrm{CH}$ and GW. However, other parameters such as Received Signal Strength (RSS) or the load of the candidate $\mathrm{GW}$ (i.e. a cluster member candidate to be elected as a $\mathrm{GW}$ ) are not accounted for in this scheme.

Decentralized vehicles clustering and gateway management scheme has been proposed in [3], and extended in [4]. In both papers, a hybrid architecture integrating VANETs with UMTS network is described. The proposed adaptive mobile GW management scheme consists of three mechanisms: multi-metric mobile GW selection, GW handover, and GW discovery/advertisement. The GW selection mechanism aims at selecting minimum number of appropriate GWs to achieve optimal communication for the whole cluster with UMTS network. The selection of GW is based on the so-called simple additive weighting technique that uses the following metrics: direction, mobility speed of $\mathrm{CH}$, its UMTS RSS, and the link (route) stability with the Source Vehicles (SVs). This latter metric is expressed by the Link Expiration Time (LET) and Route Estimation Time (RET), respectively [9]. By using LET, the predicted time of connection between two vehicles can be derived based on their motion parameters. The parameter RET expresses the minimum value of LET if more than one hop separate the SV and the $\mathrm{CH}$. Moreover, the trend of RSS based on the vehicle's movement towards or from NodeB (base station in UMTS) is also taken into account and, therefore, future levels of RSS can be predicted. However the prediction based on the direction of movement towards/from NodeB only cannot accurately describe future levels of RSS, especially in dense urban areas. Concurrently, NodeBs are not deployed on the roadside and the signal level received by vehicles may fluctuate very rapidly in a given region [10].
The main problem of the above discussed algorithms is that they focus on the technical integration of cellular networks with VANETs, and neglect the incurred cost of connection via cellular networks and their related congestion issue caused by hundreds or thousands of communicating autonomous self-driving smart cars.

To deal with this issue, a mechanism aiming at achieving a balance between VANETs and LTE resources usage is proposed in [11]. In this paper, a hybrid network architecture combining the advantages of LTE networks with VANETs is presented. In this architecture, a QoS dependent GW selection algorithm has been designed, and which considers different traffic classes, each with different QoS requirements. This algorithm consists in electing the most appropriate GW for the source vehicles and for a specific traffic class. Moreover, it takes into account the following parameters for the $\mathrm{CH}$ selection: the best RSS of SV, the load of the base station, the load of $\mathrm{CH}$, SV to $\mathrm{CH}$ link connectivity duration, as well as its load, in addition to RSS and link connectivity duration of other candidate GWs within the same cluster. Although this algorithm makes use of VANETs resources and alleviates the burden on LTE networks, it does not ensure an optimal exploitation of these cost-free resources provided by the RSUs deployed on the roads. This is mainly due to the lack of any predictive mechanism that allows a vehicle to plan its route from departure point to the destination, such that VANETs resources usage is maximized as long as it ensures the required QoS level for some applications or classes of traffic.

In this paper, we will improve the above algorithm by designing novel mechanism that adjusts the vehicles' route to ensure the highest Signal to Interference and Noise Ratio (SINR) and, thus, higher QoS provided via RSUs with respect to the maximum travel delay tolerated by the driver. This will certainly lead to maximizing the usage of VANETs resources while minimizing the usage of the relatively costly LTE-A resources. Notice that the updated vehicle route is constrained by the tolerated prolongation of journey value set by the driver. To ensure sufficient signal quality based route selection, our algorithm exploits the database called Longterm Average SINR Map (LASM) which indicates the highest signal quality in every point of the road map.

The database storing the strongest measured signal levels has been also used in [12] where each vehicle has its own database of past signal levels. Since the database is not shared, this proposal is thus suitable only for public transportation system with predefined routes, and cannot be applied to other categories of vehicles. To circumvent this limitation, our algorithm is designed based on a shared database as explained, in detail, in next section.

\section{PROPOSED AlgorithM}

This section provides a detailed description of the proposed Signal Quality-based Route Selection Algorithm (SQRSA). SQRSA aims at maximizing the utilization of RSUs resources against the LTE-A connection as long as the quality of connection required for on-board users is preserved. For this purpose, the shared signal level database called Long-term Average SINR Map and denoted as LASM is used. Based on 
the information extracted from it, the route that maximizes the utilization of RSUs' resources is selected. This route depends also on the willingness of users to slightly prolong their journey in exchange of cost-free connection with sufficient QoS provided via RSUs. This is mainly interesting for users who want to stay connected while traveling, but reduce the connection cost as much as possible. Thus, SQRSA can serve, for example, tourists, driving their own or rented cars, who want to enjoy free Internet connection while visiting the city. Moreover, our proposal can bring a paramount benefit to mobile operators. The SQRSA will lead to more efficient avoidance of cellular networks overloading, and significantly improve the offloading efficiency of LTE-A data connection since, in future cities, a substantial increase of the number of communicating cars is expected.

The rest of this section is split into three parts. First, we describe the main assumptions of the work. Second, we explain how we create, update and use the LASM information. Finally, a detailed presentation of SQRSA is given.

\section{A. Assumptions}

To ensure proper functioning of our proposal, the following set of assumptions has to be fulfilled.

First, we assume that all vehicles are equipped with GPS navigation to determine their current positions [13]. Moreover, the information from other vehicles in the vicinity or from RSUs can be used for accurate determination of the location [14]. We also assume that the destination point is known for the vehicle as well, as this will not help to search the best route only but also to avoid possible traffic jams on a wellknown route (e.g., on the way between the home and work, etc.).

Further we assume that in future transportation systems, smart vehicles will be equipped by WAVE interface [11] that allows them to obtain/report the current traffic information from/to other vehicles in the vicinity or from/to RSUs. However, the RSUs are not currently widely deployed along the road network, making many roads uncovered by such technology. Therefore, we also assume that an alternative reliable connection via LTE-A is needed as backup, especially in emergency circumstances such as road incidents. For onboard users, we assume that a Wi-Fi network is set up to provide Internet connection to these users' devices via VANETs or LTE-A, instead of direct connections of these mobile devices to the LTE-A. The Wi-Fi network is used since it is free of charge and the majority of mobile devices are equipped with this technology. Such concept exploiting Wi-Fi as GW is already widely used, especially in public transportation, such as in the city of Dublin [15].

LTE-A network is generally able to provide higher throughput than VANETs, however the incurred connection cost is still high which discourages users from using it if alternative cost-free connections are available (e.g. VANETs through RSUs) [16]. If the on-board users have low demands on services and RSUs exist in the vicinity, VANET can be used for transmission, leading to significant reduction of LTEA resources usage, and thus more resources will be available for other users outside the vehicle or without possibility of connection to VANET. Once a high QoS requirement occurs, the connection to LTE-A is still available as backup since the vehicle is equipped with LTE-A interface as well. Therefore, our proposed algorithm aims to use LTE-A only when VANETs are not able to serve users' QoS requirements, or in case of emergency for traffic information related messages. If no connection to VANETs is available, common traffic information packets are discarded after expiration since their priority is low. This assumption is very important especially in future smart cities, where the excessive usage of cellular networks can lead to unavailability of network connection. However, the cooperation of vehicles using our proposal can lead to efficient usage of cellular resources.

Since the best route in terms of RSUs coverage point of view may not be the fastest/shortest route that drivers are usually seeking for, the willingness of users to tolerate a slight prolongation of their journey in order to fulfill the required demands on resources at no cost is assumed. The prolongation of travel time does not necessarily mean a higher cost, in terms of money, of journey since the fastest route also may not be the shortest or the most economical (e.g. access fees to highways, etc.). The journey prolongation in our work does not necessarily mean excessively increasing the original journey time, but instead slightly prolonging its duration (i.e. maximum increase equals $25 \%$ of the shortest path duration).

\section{B. Long-term Average SINR Map}

The core of our proposed SQRSA consists in exploiting the long-term average SINR map denoted as LASM. This latter is created and updated using a similar well known approach, named fingerprints, presented for example in [17]. LASM as well as fingerprints exploit the values measured by users previously passed through some measurement points to improve the performance of future users' applications. Measurement points are virtual points regularly distributed along every road in the whole map. The distribution of these points can be different for city area and highways, and influences the frequency of reporting and consequently the accuracy of reconstruction of signal level map.

The process of constructing the LASM is described as follows. All passing vehicles measure the SINR level of all sensed RSUs in vicinity at each measurement point that they have crossed. After each measurement, the vehicles evaluate the sensed SINR levels and transmit the SINR value of the strongest RSU with its exact location of measurement through the so-called SINR measurement report to LASM. This report is a part of regular traffic information update transmitted through the periodic beacons if connection to RSUs is available, which means that SQRSA does not require any modification to VANETs protocols. In LASM database, weighted long-term average values of SINR level are calculated for each measurement point to suppress the fading and shadowing effects. The more actual SINR levels are the higher weights they have. A higher SINR in a specific measurement point indicates a higher quality of signal and, therefore, more resistance to signal quality deterioration due to shadowing caused by either other vehicles or other interference sources. 
To avoid the negative impact of weather conditions or any other predictable events on SINR level estimation accuracy, additional information can be taken into account. For example, if the reported SINR values are suddenly lower than usual, and the information from in-road sensors indicates that a rain has fallen, current measurement values can be tagged as "rain data" and are provided only in case of rain fall. An overview of our proposed system architecture is given in Fig. 1.

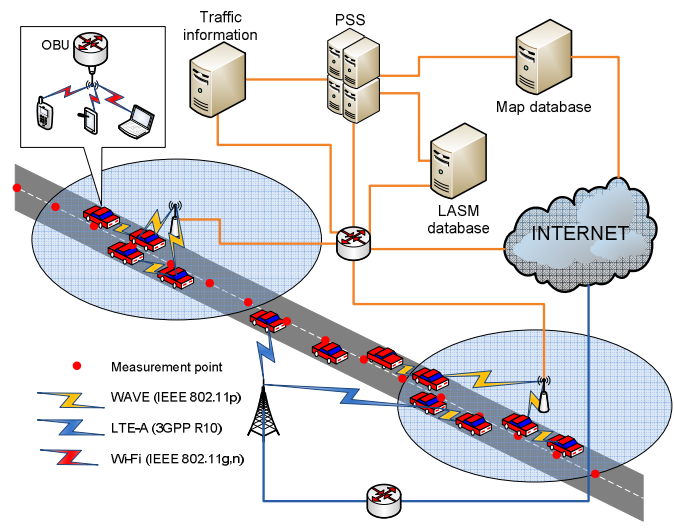

Fig. 1. System architecture overview

By combining the measured SINR with the actual positions of other vehicles, the real throughput can be computed and the selection of a route can be more efficient. However, the study of vehicles density impact on the accuracy of the estimated throughput is out of scope of this paper, and will be deeply discussed in our future work. Moreover, the assessment of the incurred cost, in terms of money spent, for a longer route compared to the corresponding connection cost of the original route is not carried out as well.

\section{Signal Quality-based Route Selection Algorithm (SQRSA)}

The computation of the best possible route in terms of maximization of the signal quality provided by RSUs is performed in dedicated Path Selection Server (PSS). The main reason of adopting remote computation tool is the reduction of the overhead associated with the transfer of huge amount of data related to LASM towards the vehicles. Moreover, using the PSS will allow us to take advantage of the available realtime traffic information to ensure more accurate results in terms of travel time estimation to the destination point.

The detailed functioning of SQRSA is described as follows. In the first step, the vehicle sends the coordinates of its current and destination locations (i.e. $C=\left(x_{C}, y_{C}\right)$ and $D=$ $\left(x_{D}, y_{D}\right)$, respectively) to the PSS. Based on current and destination points, $C$ and $D$, and using actual traffic information (i.e. congestion level, weather conditions, etc.), the PSS calculates the fastest possible path $P_{C D \_M I N}$ with the duration $t_{C D \_M I N}$. Based on the comparative study of route selection algorithms provided in [18], dynamic A* algorithm [19] can be used for selecting the fastest route. The route with the travel time $t_{C D \_M I N}$ is then sent back to the vehicle.

Based on the drivers/users willingness of prolonging their journey to get longer time of free connection via RSUs, they set the maximum tolerated prolongation of journey $t_{T P J}$ to their desirable value. The time $t_{T P J}$ can be set either directly as a fixed value, or as a prolongation ratio in percentage, or as required time to reach the destination. However, according to the user setting, the maximal tolerated time length of journey $t_{C D \_M A X}$ is computed and sent back to the PSS. This latter then selects the set of routes $\Omega_{C D \_M A X}$ which meet the following condition, among all the available routes between $C$ and $D$ (denoted as $\Omega_{C D \_A L L}$ ):

$$
\Omega_{C D_{-} M A X}=\left\{P_{k} \in \Omega_{C D_{-} A L L} \mid t_{P_{k}}<t_{C D_{-} M A X}\right\}
$$

Where $t_{P_{k}}$ is the total time length of the route $P_{k}$. Each route $P_{k}$ in the set $\Omega_{C D \_M A X}$ represents the set of measurement points denoted as $\tau\left(x_{m}, y_{m}\right)$.

$$
P_{k}=\left\{\tau\left(x_{1}, y_{1}\right), \ldots, \tau\left(x_{m}, y_{m}\right), \ldots, \tau\left(x_{z}, y_{z}\right)\right\}
$$

Where $\left(x_{m}, y_{m}\right)$ are the coordinates of the $m$-th measurement point and $z$ is the number of measurement points on the route $P_{k}$. Since the measurement points are deployed regularly, $z$ refers to the physical length of the route and it can be different for each route. Each element $\tau\left(x_{m}, y_{m}\right)$ of $P_{k}$ contains the average SINR level of the strongest RSU in coordinates $(x, y)$. These values are assigned to the individual $\tau\left(x_{m}, y_{m}\right)$ from LASM database where the signal levels for each coordinates $(x, y)$ are stored. Then the set $E_{k}$ can be derived from the set $P_{k}$ as follows:

$$
E_{k}=\left\{\tau\left(x_{m}, y_{m}\right) \in P_{k} \in \Omega_{C D_{-} M A X} \mid \tau_{M I N}>\tau\left(x_{m}, y_{m}\right)\right\}
$$

Where $\tau_{M I N}$ is the minimum tolerated SINR. This value is set to $5 \mathrm{~dB}$ according to [20]. The set $E_{k}$ contains the measurement points of the $k$-th route that do not satisfy the condition on minimum SINR level. This implies that the route with sufficient SINR level provided via RSUs is the route which has the minimum number of elements in the set $E_{k}$. In other words, this route (i.e. the best route) respects the maximum tolerated increase in travel time set by the user and has the shortest part of the journey without sufficient SINR level through RSUs. The selected best route in terms of signal quality provided via RSUs is then sent back to the vehicle. A flowchart summarizing the main steps of the proposed algorithm is shown in Fig. 2.

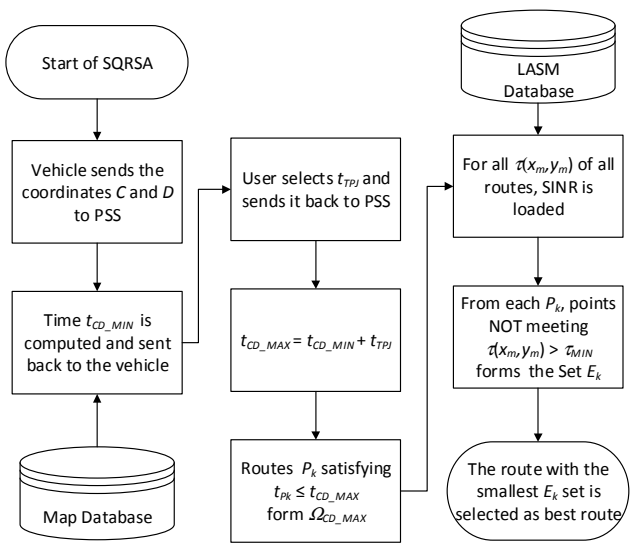

Fig. 2. The main steps of the proposed SQRSA 


\section{Simulation Settings AND ScEnarios}

To validate the effectiveness of the proposed algorithm, extensive simulations were conducted using MATLAB. As simulation scenario, a map with 10 perpendicular streets with total length of 60 kilometers $(5 \times 5 \mathrm{~km} ; 5 \times 7 \mathrm{~km})$ is used. Every street is composed by 4 road segments which are bounded by two adjacent intersections. Each road segment has different traffic flow parameters which determine the time needed for a vehicle to cross it, and which are updated at every simulation run. This means that the time needed for crossing two streets with the same physical length (or the same street but in different simulation runs) can be different. The speed in each road segment varies between $3 \mathrm{~km} / \mathrm{h}$ and $60 \mathrm{~km} / \mathrm{h}$ with an average speed of $30 \mathrm{~km} / \mathrm{h}$.

In the map below, eNBs and RSUs are deployed. While the number and positions of eNBs are fixed, the number of RSUs is determined by the simulation scenario and their positions are randomly changed at every simulation run. The fixed number and positions are used to eliminate the impact of road environments change between the different simulation runs since the focus of this paper is on RSUs. An example of simulation scenario is depicted in Fig. 3.

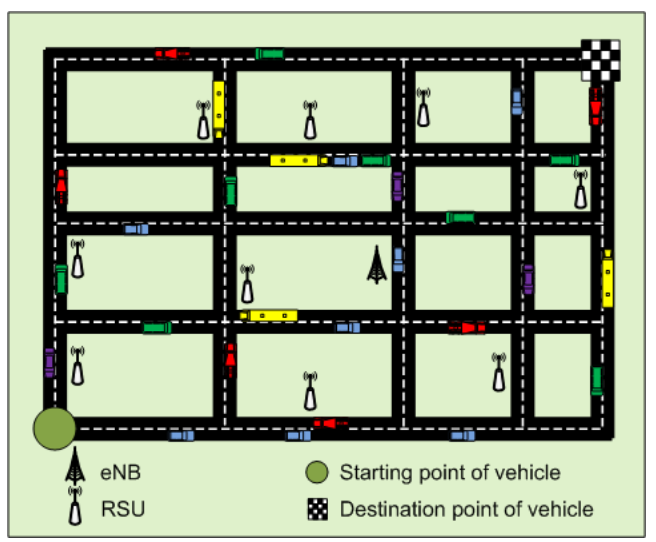

Fig. 3. Simulation environment

Since the street parameters as well as positions of RSUs vary in every simulation run, the starting and destination points of vehicles are fixed for all the runs. The departure point of every vehicle is located in the intersection on the bottom left (denoted as green circle in Fig. 3) while its destination point is located in the intersection on top right (denoted as check flag in Fig. 3). Based on the users' preferences, the appropriate route selection algorithm will be chosen and launched to search for the requested route.

In our simulation, the path loss of WAVE is modeled as a combination of free space path loss model with Two-Ray Ground path loss model as suggested in [21]. The SINR level is derived as described in [20] for all measurement points which are placed in every 1 meter of each street. As sufficient quality of signal, SINR higher than $5 \mathrm{~dB}$ is set. If SINR is lower than this threshold, the signal is considered as unusable.

For LTE-A, Okumura-Hata path loss model for urban scenarios [22] is used. The quality of signal for LTE-A users is derived according to [23]. Since the time needed for reconnection between VANET and LTE-A (and vice versa) is negligible in comparison with the connection time to VANET or LTE-A, it is not considered in our simulation results. Other major simulation parameters are listed in TABLE I.

TABLE I. SIMULATION PARAMETERS

\begin{tabular}{|l|r|}
\hline \multicolumn{1}{|c|}{ Parameters } & \multicolumn{1}{c|}{ Value } \\
\hline Frequency band WAVE/LTE-A [GHz] & $5.9 / 2$ \\
\hline Frequency bandwidth WAVE/LTE-A [MHz] & $10 / 20$ \\
\hline Transmission power of RSUs/eNBs [dBm] & $20 / 23$ \\
\hline Height of RSUs/eNBs [m] & $1.8 / 32$ \\
\hline Minimum distance between two RSUs [m] & 500 \\
\hline Number of RSUs/eNBs & $\{10-70\} / 4$ \\
\hline Modulation/code rate of WAVE & BPSK / 0.5 \\
\hline Noise level WAVE/LTE-A [dBm] & -95 \\
\hline SINR outage level [dB] & 5 \\
\hline Average speed in street segment [km/h] & 30 \\
\hline Total distance between start and destination point [km] & 12 \\
\hline Number of simulation runs & 100 \\
\hline
\end{tabular}

\section{Performance Evaluation}

In this section, the performance of our proposed algorithm SQRSA is evaluated and compared to other journey planning approaches presented in literature. This section is split into three subsections as described below. First, the three major journey-planning approaches are discussed. Then, the chosen performance evaluation metrics are introduced. Finally, the obtained simulation results are presented and analyzed in detail.

\section{A. Compared Algorithms}

In simulation, there are two different types of users who consider different levels of service and three different journeyplanning approaches.

The first type of users consists of those who prefer to drive through the route that ensures the longest possible connection with the highest quality of connection, regardless of their real requirements and the incurred cost [2]. This user type is denoted as Highest Quality (HQ). Due to the higher requirements of these users, they use primarily LTE-A network and connection to RSUs is used only if no better eNBs connection is available. For the sake of clarity, only connection to eNBs is considered in the results, meaning that although HQ users can exploit RSUs resources to extend their connection time; this fact is not depicted in the results for comparison purposes.

The second type of users is those who prefer the route with the longest possible connection, and which ensures sufficient connection quality with respect to the resulting price of connection. Such users exploit the fact that the connection via VANETs is free of charge and they can use it instead of LTEA. A user in this group is denoted as Sufficient Quality (SQ). This user is looking for the route that provides the longest connection to RSUs with sufficient signal quality and LTE-A is used only in locations where no sufficient signal quality provided by RSUs is available. Similarly to HQ user type, in simulation results only connection to RSUs is considered, meaning that if no satisfactory VANETs connection is available, users connect to LTE-A as backup, however this connection is not depicted in the results. 
For comparison purposes, three different journey-planning approaches are used: planned journey with respect to the $\mathrm{HQ} / \mathrm{SQ}$, unplanned journey with respect to the HQ/SQ and the fastest journey regardless of HQ or SQ. By using planned journey based approach, the best route from departure point to destination point is selected according to the best parameters of the whole route. Planned journey used by SQ user corresponds to our proposal and is denoted as SQRSA in the plotted results. Similarly, analogous principle can be also used by HQ users to plan their journey. In this case, the acronym HQRSA (Highest Quality based Route Selection Algorithm) is used in the plotted results.

On the other hand, by using unplanned journey, the users decide about their next direction when they reach an intersection as described in 0 . This means that the best route is composed by the set of road segments chosen as the best possible options at each intersection, one by one, and not based on the best end-to-end route. For the decision, the LASM of eNBs/RSUs is used as well. However, such unplanned journey can lead to the infinite loop problem. Therefore, we assume that every next intersection has to be geographically closer to the destination point than the intersection in which the decision is made, which eliminates the above problem. Unplanned algorithms used by SQ and HQ users are denoted as uSQ and uHQ in the depicted results, respectively.

The Fastest Route (FR) approach is a special category of the planned journey approach. In FR, users aim only to drive through the route that minimizes their travel time and they are not willing to tolerate a prolongation of their journey for any reason. This travel time corresponds to time $t_{C D \text { MIN }}$ derived for SQRSA in section III.C. Therefore, the travel time of the journey in this case is always the same for both HQ and SQ users, whereas the HQ and SQ coverage is different. These users plan their journey using dynamic A* algorithm [19].

In our simulation, we also evaluate the impact of RSUs deployment density (i.e., 10, 40, and 70 RSUs deployed on the road network) on the efficiency of the simulated algorithms. On the other hand, the number of LTE-A eNBs is fixed for all simulation runs and, therefore, the obtained results for both HQRSA and uHQ are similar as their performances do not depend on the number of RSUs in the simulated area.

\section{B. Performance Metrics}

In our simulation, the performance of the above-discussed algorithms is evaluated based on the following four key parameters.

\section{1) RSUs/eNBs coverage}

RSUs/eNBs coverage $\Phi$ represents the ratio of measurement points covered by RSUs or eNBs to the total number of measurement points $z$ that the route $k$ selected by using given algorithm contains. RSUs/eNBs coverage for a particular Type of User (TU) (i.e. HQ or SQ) can be expressed as:

$$
\Phi_{k}^{T U}=\frac{\pi_{k}^{T U}}{z_{k}} \cdot 100 \%
$$

Where $\pi_{k}^{T U}$ refers to the route $k$ length in terms of number of measurement points where the user is connected to RSUs/eNBs with sufficient/highest QoS, and $z_{k}$ is the total length of this route $k$. Note that:

$$
z_{k}=\pi_{k}+E_{k}
$$

Where $E_{k}$ represents the number of measurement points, where no sufficient RSUs/LTE-A connection is ensured as shown in (3).

\section{2) Coverage improvement}

Coverage improvement $\Theta$ refers to the increase of RSUs/eNBs coverage level reached by using a particular algorithm compared to the FR approach. The coverage improvement for a particular type of user TU can be expressed as:

$$
\Theta_{k}^{T U}=\frac{\Phi_{k}^{T U}-\Phi_{F R}^{T U}}{\Phi_{F R}^{T U}} \cdot 100 \%
$$

Where $\Phi_{F R}^{T U}$ is the percentage of coverage reached by using FR approach.

\section{3) Prolongation efficiency}

Prolongation efficiency $\Psi$ represents the achieved improvement in coverage level of the real journey time prolongation compared to that achieved using FR approach.

The prolongation efficiency ratio for a particular type of user TU can be expressed as:

$$
\Psi_{k}^{T U}=\frac{\Theta_{k}^{T U}}{\vartheta_{k}^{T U}}
$$

Where $\vartheta_{k}^{T U}$ denotes the prolongation ratio of the user travel time expressed as:

$$
\vartheta_{k}^{T U}=\frac{t_{P_{k}}-t_{C D_{-} M I N}}{t_{C D_{-} M I N}} \cdot 100 \%
$$

\section{4) LTE-A offloading improvement}

LTE-A offloading improvement ratio $\Xi$ expresses the percentage of the additional LTE-A offloading brought by our proposal based on the Real Prolongation of Travel Time (RPTT), compared to that achieved using FR approach. This ratio can be calculated by the following formula:

$$
\Xi_{k}=\left(\frac{\Phi_{k}^{\text {both }}}{\Phi_{k}^{L T E-A}}-\frac{\Phi_{F R}^{\text {both }}}{\Phi_{F R}^{L T E-A}}\right) \cdot 100 \%
$$

Where $\Phi_{F R}^{\text {both }}$ and $\Phi_{k}^{\text {both }}$ refer to the percentage of measurement points covered by using both networks LTE-A and VANETs, while $\Phi_{F R}^{L T E-A}$ and $\Phi_{k}^{L T E-A}$ refer to percentage of measurement points covered by LTE-A connection only. 


\section{Simulation Results}

In this subsection, we plot and discuss the obtained simulation results of the above-described scenarios.

\section{1) RSUs/eNBs coverage}

In Fig. 4, the achieved RSUs/eNBs coverage for the users' journey route based on the RPTT is presented. These results show that SQRSA exhibits significant improvement of the coverage ensured by RSUs with a relatively low prolongation of the travel time, as compared to FR SQ and USQ algorithms under varying RSUs deployment density. The attained coverage level increases with the increase of RSUs density and reaches its highest values with 70 deployed RSUs. The route coverage by free connection to RSUs can be improved significantly by selecting slightly longer routes, compared to the FR, under low density of RSUs, while under higher densities a similar coverage to FR is achieved by SQRSA but at lower prolongation of journey as compared to the previous case. Notice that LTE-A coverage improvement can be also achieved by HQRSA.

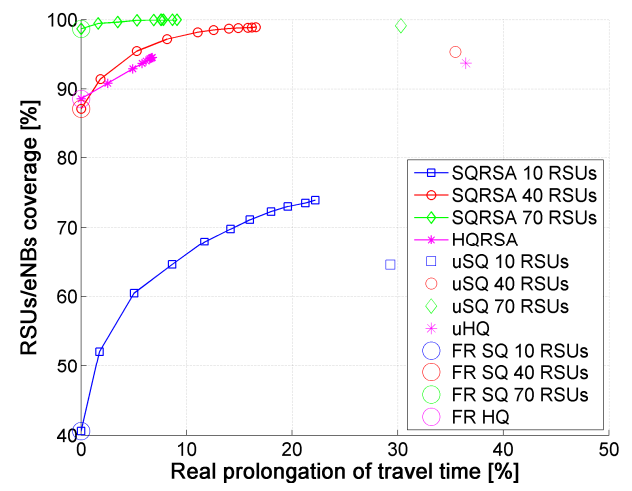

Fig. 4. Extension of RSUs/eNBs coverage vs. RPTT

On the other hand, the coverage level is also improved using unplanned approaches (uSQ or uHQ) however the required prolongation of travel time is significant in this case.

\section{2) Coverage improvement}

The coverage improvement achieved by each algorithm is depicted in Fig. 5 from which we observe that the highest improvement, in comparison with the results of fastest route, are obtained by SQRSA algorithm in case of 10 deployed RSUs only. For example, a $22 \%$ of RPTT can lead to an extension of RSUs coverage on route by up to $82 \%$. However, this improvement decreases with the rise in RSUs density, leading to very marginal enhancement in case of 70 deployed RSUs (e.g. for $9 \%$ of RPTT, the coverage is improved by $1.3 \%$ only).

Under low RSUs deployment density on the map, the probability that the fastest route is also the route with the best coverage providing sufficient signal quality is very low. In contrast, a highly dense deployment of RSUs will approximately cover all the places in the area. This coverage level will then ensure sufficient signal quality providing sufficient signal quality, and there is almost no place for another improvement of coverage by selecting an alternative route.

Note that the unplanned approaches also improve the coverage, however, similar to Fig. 4 results, the RPTT needed by UHQ and USQ is very high compared to that required by HQRSA and SQRSA (under all scenarios) to achieve a significantly higher improvement.

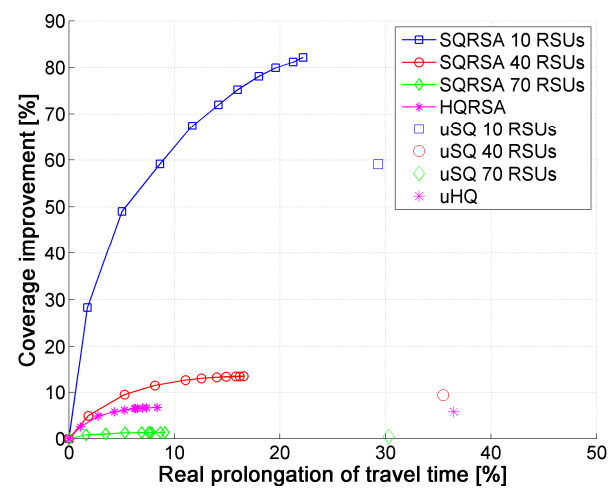

Fig. 5. RSUs/eNBs coverage improvement vs. RPTT

\section{3) Prolongation efficiency}

In Fig. 6, the travel time prolongation efficiency ratio defined in (7) is depicted. The plotted results prove previous results regarding the coverage improvement efficiency when various densities of RSUs are considered. These results highlight that the prolongation efficiency decreases with the increase in the number of RSUs, and is inversely proportional to the RPTT values. For example, for SQRSA with 10 RSUs, the efficiency ratio for first prolongation is greater than 16; while the efficiency ratio of the same algorithm in case of 70 RSUs is lower than 0.5 .

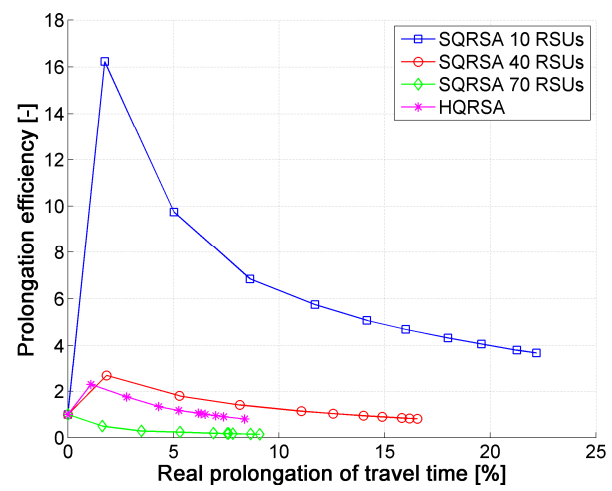

Fig. 6. RSUs/eNBs coverage improvement vs. RPTT

\section{4) Efficiency of prolongation of travel time}

The main purpose of our proposal is to offload LTE-A network by selecting alternative route with higher RSUs coverage and ensuring sufficient signal quality to fulfill the users' requirements.

Fig. 7 shows the offloading improvement ratio of our SQRSA algorithm and uSQ algorithm under varying RSUs 
densities. As the results show, the highest improvement ratio is achieved under lower RSUs density. A real travel time prolongation of $22 \%$ can lead to LTE-A network offload of more than $32 \%$. With the increase of the deployed RSUs in the simulation scenario, the LTE-A network can naturally offloaded by those RSUs and, therefore, the impact of the journey prolongation becomes less significant. As the results show, for 70 deployed RSUs in the simulation, the improvement of LTE-A offloading is nearly negligible.

As discussed above, for an unplanned journey, the offloading can be still feasible; however, by applying such algorithm, an improvement equals to $70 \%$ of the offloading improvement achieved by SQRSA can be achieved, under all the scenarios, with a significant increase in RPTT.

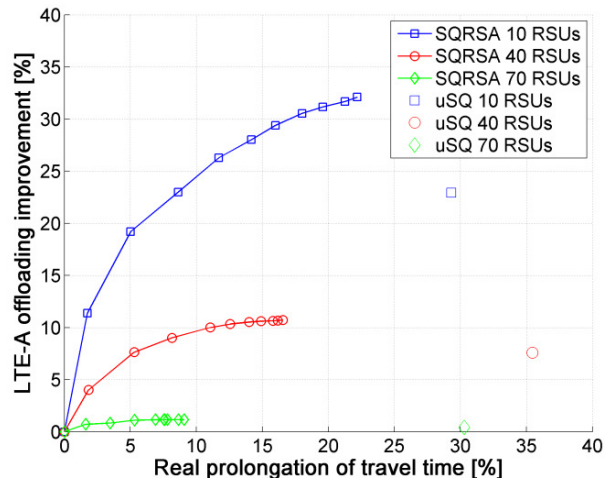

Fig. 7. LTE-A offloading improvement

\section{CONCLUSION}

In this paper, we have proposed new route selection algorithm, named SQRSA, offering sufficient quality of signal for drivers and passengers applications via VANETs connection through RSUs. The proposed algorithm ensures the selection of a route, relaying the departure and destination points of the vehicle, which offers sufficient SINR for onboard users. The maximum length of this route is limited by the users' willingness to tolerate longer journey time in exchange of free connection through VANET. As the simulation results show, our proposal outperforms the standard fastest route algorithm as well as other literature schemes. These results reveal that our algorithm is especially suitable for road networks with a limited number of deployed RSUs, in which very high efficiency can be achieved. By using SQRSA, the offloading of LTE-A can be improved by almost $20 \%$ under a journey time prolongation equals to $5 \%$ only. In our future work, we will extend the proposed algorithm to consider the available connection time to RSUs and design a mechanism that enables more efficient utilization of LTE-A resources.

\section{ACKNOWLEDGMENT}

This work was supported, in part, by Science Foundation Ireland grant 10/CE/I1855 to Lero - the Irish Software Engineering Research Centre (www.lero.ie), and by the grant of Czech Technical University in Prague No. SGS SGS13/199/OHK3/3T/13.

\section{REFERENCES}

[1] K. Zhu, et al. "Mobility and handoff management in vehicular networks: a survey." Wiley WCMC journal 11.4 (2011): 459-476.

[2] G. Rémy, et al. "Lte4v2x: Lte for a centralized vanet organization." In IEEE GLOBECOM 2011, 2011.

[3] T. Taleb, et al. "Design guidelines for a network architecture integrating vanet with $3 \mathrm{~g}$ \& beyond networks." In IEEE GLOBECOM 2010, 2010.

[4] A. Benslimane, et al. "Dynamic clustering-based adaptive mobile gateway management in integrated VANET-3G Heterogeneous Wireless Networks." In IEEE JSAC, 29.3 (2011): 559-570.

[5] Google Auto, http://www.android.com/auto/ Web. July. 2014.

[6] Open Automotice Aliance, http://www.openautoalliance.net/. Web. July. 2014.

[7] Ch. Urmson, "Just press go: designing a self-driving vehicle", http://googleblog.blogspot.cz/2014/05/just-press-go-designing-selfdriving.html May 2014. Web. July. 2014.

[8] L. CH. Tung, et al. "Priority-Based Congestion Control Algorithm for Cross-Traffic Assistance on LTE Networks." IEEE VTC Fall 2013.

[9] A. Benslimane, et al. "An efficient routing protocol for connecting vehicular networks to the Internet." Pervasive and Mobile Computing 7.1 (2011): 98-113.

[10] S. A. Hosseini Tabatabaei, et al. "Improving propagation modeling in urban environments for vehicular ad hoc networks." Intelligent Transportation Systems, IEEE Transactions on 12.3 (2011): 705-716.

[11] G. E. M. Zhioua, et al. "A multi-metric qos-balancing scheme for gateway selection in a clustered hybrid vanet network." IEEE WiMob 2012.

[12] W-K. Lai, et al. "A novel bandwidth prediction and queue management scheme for public transportation systems," Global Mobile Congress (GMC), 2011, vol., no., pp.1,6, 17-18 Oct. 2011.

[13] N. Alam, et al. "Range and range-rate measurements using DSRC: facts and challenges." Proc. of IGNSS Symp. 2009.

[14] N. Alam, et al. "Relative positioning enhancement in VANETs: A tight integration approach." Intelligent Transportation Systems, IEEE Transactions on 14.1 (2013): 47-55.

[15] J. Kennedy, "First of new fleet of $80 \mathrm{Wi}-\mathrm{Fi}$ and CCTV-enabled buses begin rolling onto Dublin streets" siliconrepublic.com N.p., 11 October 2012. Web. July. 2014.

[16] Notice of proposed rulemaking and order FCC 02-302. Federal Communications Commission, November 2002.

[17] A. Prasad, et al. "Energy Efficient Small-Cell Discovery Using Received Signal Strength Based Radio Maps," in IEEE Vehicular Technology Conference 2013 (VTC Spring 2013), Dresden, July 2013.

[18] S. Wang, et al. "Comprehensive performance analysis and comparison of vehicles routing algorithms in smart cities." Global Information Infrastructure Symposium, 2013. IEEE, 2013.

[19] P. E. Hart, et al."A formal basis for the heuristic determination of minimum cost paths." Systems Science and Cybernetics, IEEE Transactions on 4.2 (1968): 100-107.

[20] Y. Zang, et al. "An error model for inter-vehicle communications in highway scenarios at $5.9 \mathrm{GHz}$." Proceedings of the 2nd ACM international workshop on Performance evaluation of wireless ad hoc, sensor, and ubiquitous networks. ACM, 2005.

[21] Ch. Sommer, et al. "On the applicability of Two-Ray path loss models for vehicular network simulation." VNC. 2012

[22] J. S. Seybold, Introduction to RF propagation. John Wiley \& Sons, 2005.

[23] 3GPP TS 36.213, "Evolved Universal Terrestrial Radio Access (EUTRA); Physical layer procedures," Mar. 2014.

[24] L. Fu, et al. "Heuristic shortest path algorithms for transportation applications: state of the art." Computers \& Operations Research 33.11 (2006): 3324-3343. 